\title{
Data-Driven Energy Efficient Predictive Resource Allocation in Internet of Vehicles
}

\author{
Na Xue*, Haixia Zhang*†, Chuanting Zhang ${ }^{\ddagger}$, Tiantian $\mathrm{Li}^{*}$, Dongfeng Yuan* \\ *Shandong Key Laboratory of Wireless Communication Technologies, Shandong University, Jinan, China \\ ${ }^{\dagger}$ School of Control Science and Engineering, Shandong University, Jinan, China \\ ${ }^{\ddagger}$ Computer, Electrical and Mathematical Science and Engineering Division, \\ King Abdullah University of Science and Technology, Thuwal, Saudi Arabia \\ Email: na.xue7@mail.sdu.edu.cn, haixia.zhang@sdu.edu.cn
}

\begin{abstract}
In Internet of Vehicles (IoV), the high mobility of vehicles aggravates the uneven and dynamic spatial-temporal distribution of wireless traffic, leading to low resource utilization. To improve the wireless resource utilization efficiency of $\mathrm{IoV}$, this paper investigates predictive resource allocation strategy by exploiting vehicle mobility information. To characterize vehicle's speed distribution, we adopt a kernel density estimation method to analyze the real trajectory dataset. Based on this analysis, we propose an iterative predictive resource allocation scheme considering different mobility patterns and channel distribution information. Simulation results demonstrate that our proposed scheme converges well and can obtain considerable performance gains over non-predictive resource allocation schemes.
\end{abstract}

Index Terms-Energy efficiency, predictive resource allocation, data-driven, Internet of Vehicles (IoV).

\section{INTRODUCTION}

As one of the typical application scenarios of the fifth generation (5G), Internet of Vehicles (IoV) has attracted tremendous attention not only from academia but also from industry in recent years [1]. IoV provides information exchange via vehicle-to-vehicle (V2V), vehicle-to-infrastructure (V2I) and other vehicle-to-x (V2X) communications. However, the high mobility of vehicles makes the spatio-temporal distribution of wireless traffic uneven and dynamic. In such scenarios, conventional resource allocation (RA) schemes that have no aware of future variation of traffic demands lead to extremely low resource utilization efficiency. To tackle this problem, predictive resource allocation (PRA) scheme, which benefits from predicting the user mobility and traffic variation accurately through big data analysis and deep learning [2]-[4] before resource allocation, has been proposed and recognized as one of the most promising ways [5]-[7].

PRA can make a long term resource allocation by using the prior information and has shown remarkable gain in energy savings and pervasive QoS satisfaction [7]-[9]. For instance, the authors in [7], [9] investigated how to allocate the time resources in advance to reduce the energy consumption while satisfying users' QoS. In [7], the authors investigated how to exploit the prediction of user data rates to minimize the total required transmission time. With a fixed transmission power of base station (BS), minimizing the required transmission airtime is equivalent to minimizing energy consumption. To deal with prediction uncertainty, a stochastic PRA framework
[9] using robust optimization was proposed, where a low complexity heuristic algorithm with the Bernstein approximation and particle filter was used to allocate time resource. Note that these works only focus on time resource allocation. To further make use of energy resource, the authors in [8] proposed a two-timescale heuristic policy where a coarse resource allocation plan was made in large timescale and a fine power water-filling scheme was executed in small timescale. However, there still exist some challenges that need to be dealt with. On the one hand, the aforementioned works ignore the real mobility patterns of user equipment. On the other hand, the fast time-varying channel state information (CSI) in $\mathrm{IoV}$ is hard to estimate accurately before resource allocation. Motivated by these points, how to design an efficient PRA scheme considering both real mobility patterns of vehicle and imperfect CSI is worth investigating.

In this work, we propose an energy efficient predictive resource allocation (EE-PRA) strategy with data-driven mobility pattern in IoV. To explore the real mobility patterns of vehicle, we perform data analysis by using real world dataset and extracting the speed information of different roads. Then we adopt a kernel density estimation method to fit the speed distribution of vehicles. An energy efficiency maximization problem with different mobility patterns of vehicle user equipment (VUE) is formulated. To solve this problem, we derive an approximation of average data rate from the outage probability (OP) by utilizing channel distribution information (CDI). Then, an iterative EE-PRA algorithm based on Dinkelbach method is proposed. Simulation results show that compared with non-predictive resource allocation schemes, our proposed scheme with perfect and imperfect prediction can both significantly improve the performance of energy efficiency.

The remainder of this paper is organized as follows. In Section II we introduce the data analysis and system model. In Section III, an iterative algorithm for PRA is proposed. Simulation results are presented in Section IV and we conclude this work in Section V.

\section{Data Analysis And System Model}

In this section, we first perform big data analysis on the real dataset and adopt function estimation to fit the vehicle 


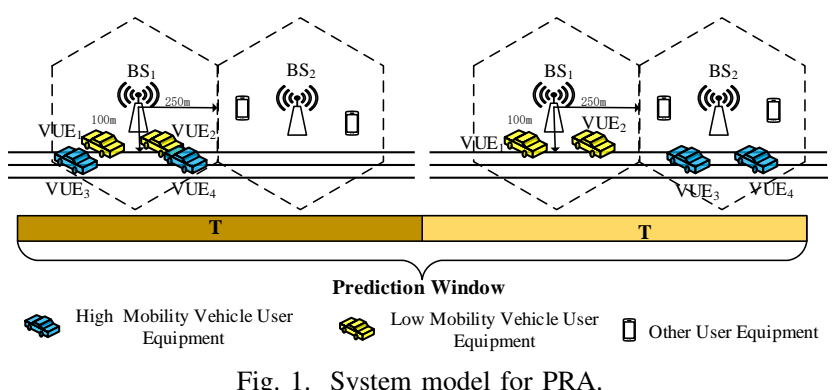

Fig. 1. System model for PRA.

speed distribution. Inspired by the results of data analysis, we consider a two-cell system as depicted in Fig. 1, where there is a central processor $(\mathrm{CP})$ that can gather data from BSs and VUEs to predict future VUEs mobility and feedback results to corresponding entity.

\section{A. Speed Distribution Analysis}

There is a large amount of data in IoV. To explore the mobility feature of VUEs, we analyze the T-drive Data, which is a public dataset from Microsoft Research Asia [10]. This dataset contains GPS trajectories of 10357 taxis in one week, from $02 / 02 / 2018$ to $02 / 08 / 2018$. Each record in this dataset contains the taxi id, data time, longitude and latitude. We choose one specific road and collect all the records of vehicles that pass through this road. Then, we plot the histogram of the speed distribution and try to estimate its probability density function. It is noted that function estimation includes parameter estimation and non-parametric estimation. We first perform parameter estimation of exponential distribution and normal distribution based on histogram. Then, one of the typical nonparametric estimations, i.e., kernel density estimation (KDE) [11] is exploited. In this paper, we adopt one of the KDE funcitons, i.e., the KDE-gaussian, which uses gaussian kernels to interpolate the original data. It can be expressed as

$$
\begin{gathered}
\hat{f}(x)=\frac{1}{N} \sum_{i=1}^{N} \kappa\left(\frac{x-x_{i}}{h}\right), \\
\kappa(\mathrm{x})=\frac{1}{\sqrt{2 \pi}} \exp \left(-\frac{x^{2}}{2}\right),
\end{gathered}
$$

where $x_{i}$ and $N$ are the sample value and the number of samples respectively. $i$ is the sample index, $h$ is the bandwidth of KDE which is also the width of kernel. $\kappa(\mathrm{x})$ represents the gaussian kernel. In fact, different mobility patterns may affect system performance. This inspires us to investigate the impact of different speed distributions on the performance of PRA scheme.

\section{B. System Model}

We consider a downlink transmission system, where two BSs are located on the same side of one road along a straight line serves $K$ VUEs. The shortest distance between BS and road is $100 \mathrm{~m}$. The coverage area of each BS is $R_{b}$. The maximum transmit power and the maximum bandwidth are $P_{\max }$ and $W_{\max }$, respectively. Taking the different traffic loads between adjacent cells into account, we assume that the available bandwidth in $\mathrm{BS}_{1}$ is more than that in $\mathrm{BS}_{2}$, i.e., $W_{1}>W_{2} . K$ VUEs are divided into two groups, i.e., the low mobility vehicle user equipment (LUE) group $\mathcal{K}_{\mathcal{L}} \triangleq\left\{1,2, \cdots, K_{L}\right\}$ and the high mobility vehicle user equipment (HUE) group $\mathcal{K}_{\mathcal{H}} \triangleq\left\{1,2, \cdots, K_{H}\right\}$.

Considering the mobility of HUEs, we divide the prediction window into two phases, $j \in \mathcal{J}=\{1,2\}$. The duration of each phase is $T=\sum_{t=1}^{N_{s}} \tau$, where $N_{s}$ denotes the number of slots in one phase and $\tau$ represents the duration of one slot. In the first phase, LUEs and HUEs are both associated with $\mathrm{BS}_{1}$. In the second phase, LUEs are still associated with $\mathrm{BS}_{1}$ and HUEs are associated with $\mathrm{BS}_{2}$ due to high mobility. At the start of prediction window, each VUE sends only one videoon-demand $(\mathrm{VoD})$ request to $\mathrm{BS}_{1}$ that at least $B$ bits data is required to be transmitted within the prediction window. In non-PRA scheme, the achievable data rate is based on instantaneous CSI. While in PRA scheme, instantaneous CSI is not available in advance, so the achievable data rate is based on imperfect CSI. In this case, transmission outage event will occur when the evaluated data rate in PRA exceed the actual achievable capacity. Therefore, we can get the outage probability constraint as:

$$
\operatorname{Pr}\left(R_{k}^{j, t} \geq \mathcal{C}_{k}^{j, t}\right) \leq \varepsilon,
$$

where $R_{k}^{j, t}$ is the evaluated data rate of VUE $k$ in $t$-th slot of $j$-th phase in PRA. $\mathcal{C}_{k}^{j, t}$ is the achievable capacity with perfect CSI of VUE $k$ in $t$-th slot of $j$-th phase, which can be expressed as:

$$
\mathcal{C}_{k}^{j, t}=W_{k}^{j} \log _{2}\left(1+\frac{\alpha_{k}^{j} p_{k}^{j}\left|h_{k}^{j, t}\right|^{2}}{N_{0} W_{k}^{j}}\right),
$$

where $W_{k}^{j}$ is the available bandwidth of the associated BS. $p_{k}^{j}$ represents transmit power of BS in $j$-th phase to VUE $k$, which remains constant in one phase. $\alpha_{k}^{j}=\left(\bar{d}_{k}^{j}\right)^{-\beta} g_{k}^{j}$ is the average large scale channel gain of VUE $k$ in $j$-th phase. Here, $d_{k}^{j}$ is the average distance between VUE $k$ and associated BS in $j$-th phase, $\beta$ is the path loss exponent, and $g_{k}^{j}$ represents shadowing which follows the log-normal distribution and is i.i.d among different VUEs. $h_{k}^{j, t}$ is the small scale Rayleigh fading channel in $t$-th slot of $j$-th phase. $N_{0}$ is the the noise power spectral density. $\varepsilon$ is a parameter close to zero.

\section{Energy-Efficient Predictive Resource ALLOCATION}

In this section, an energy efficiency maximization problem restricted by QoS and OP is formulated. Due to non-convex property of the objective function and constraint, we propose an iterative algorithm based on fractional programming and CDI to solve this problem.

\section{A. Problem Formulation}

The objective of this work is to maximize energy efficiency (EE) of the system. EE is the ratio of the amount of data transmitted to the energy consumption within the specific period. While in PRA, the amount of data transmitted and 
energy consumption are random variables as the instantaneous channel gains are unknown. Besides, the total number of time slots in one phase is large enough that maximizing the above $\mathrm{EE}$ is equivalent to maximizing the ratio of the average amount of data transmitted to the average energy consumption. Then, the average $\mathrm{EE}, E E_{\text {ave }}$ can be expressed as:

$$
E E_{\text {ave }} \triangleq \frac{\mathbb{E}_{h}\left(\sum_{j=1}^{2} \sum_{k=1}^{K} m_{k}^{j} \sum_{t=1}^{N_{s}} \tau R_{k}^{j, t}\right)}{\mathbb{E}_{h}\left(\sum_{j=1}^{2}\left(\sum_{k=1}^{K} p_{k}^{j} m_{k}^{j}+P_{c i r}\right) T\right)},
$$

where $m_{k}^{j}$ is the allocated time ratio in one phase. We define the average data rate of VUE $k$ in $j$-th phase as $\bar{R}_{k}^{j}=$ $\frac{\mathbb{E}_{h}\left(\sum_{t=1}^{N_{s}} \tau R_{k}^{j, t}\right)}{T}$. Then the optimization problem is formulated as:

$$
\begin{array}{ll}
\max _{\mathbf{m}, \mathbf{p}} & \frac{\sum_{j=1}^{2} \sum_{k=1}^{K} \bar{R}_{k}^{j} m_{k}^{j}}{\sum_{j=1}^{2}\left(\sum_{k=1}^{K} p_{k}^{j} m_{k}^{j}+P_{c i r}\right)} \\
\text { s.t. } & \sum_{j=1}^{2} \bar{R}_{k}^{j} m_{k}^{j} T \geq B, \quad \forall k \in \mathcal{K}, \\
& \operatorname{Pr}\left(R_{k}^{j, t} \geq \mathcal{C}_{k}^{j, t}\right) \leq \varepsilon, \quad \forall j, \quad \forall t, \quad \forall k \in \mathcal{K}, \\
& \sum_{k=1}^{K} m_{k}^{1} \leq 1, \quad \forall k \in \mathcal{K}, \\
& 0 \leq m_{k}^{j} \leq 1, \quad \forall j, \quad \forall k \in \mathcal{K}, \\
& 0 \leq p_{k}^{j} \leq \frac{W_{k}^{j}}{W_{\max }} P_{\max }, \quad \forall j, \quad \forall k \in \mathcal{K} .
\end{array}
$$

(6b) ensures that the QoS requirement of VUE $k$ is satisfied and $\mathcal{K}=\mathcal{K}_{\mathcal{L}} \cup \mathcal{K}_{\mathcal{H}}$. (6c) is the constraint of VUE's OP. (6d) means that the sum of allocated time ratio is less than 1 during the first phase. (6e) and (6f) are boundary constraints for time allocation variables and power control variables, respectively. As for the third term in (6f), $\frac{W_{k}^{j}}{W_{\max }} P_{\max }$ means the upper limit of transmit power is proportional to the available bandwidth.

It can be observed that this optimization problem is nonconvex due to the non-convexity of objective function and $\mathrm{OP}$ constraint. Focusing on the objective function in the fractional form, we define the maximum energy efficiency $\mathrm{q}^{*}$ according to the nonlinear fractional programming as:

$$
q^{*}=\max _{\mathbf{m}, \mathbf{p}} \frac{\sum_{j=1}^{2} \sum_{k=1}^{K} \bar{R}_{k}^{j} m_{k}^{j}}{\sum_{j=1}^{2} \sum_{k=1}^{K} p_{k}^{j} m_{k}^{j}+2 P_{c i r}} .
$$

Then, we have:

$$
\begin{aligned}
F\left(q^{*}\right) & =\max _{\mathbf{m}, \mathbf{p}} \sum_{j=1}^{2} \sum_{k=1}^{K} \bar{R}_{k}^{j} m_{k}^{j}-q^{*} \sum_{j=1}^{2} \sum_{k=1}^{K} p_{k}^{j} m_{k}^{j}-2 q^{*} P_{c i r} \\
& =\max _{\mathbf{m}, \mathbf{p}} N(\mathbf{m}, \mathbf{p})-q^{*} D(\mathbf{m}, \mathbf{p})
\end{aligned}
$$

which is a convex function with respect to $q^{*}$. As for the OP constraint, we use CDI, the statistical property of Rayleigh channel, to get a closed-form expression of VUE data rate. For Rayleigh fading channels, $h_{k}^{j, t} \sim \mathcal{C N}(0,1)$ is an inde- pendent complex Gaussian random variable, and $\left|h_{k}^{j, t}\right|^{2}$ has an exponential distribution with parameter 1 [12]. Hence, we have:

$$
\left.\begin{array}{rl}
\operatorname{Pr}\left(R_{k}^{j, t} \geq \mathcal{C}_{k}^{j, t}\right) & =\operatorname{Pr}\left(\left|h_{k}^{j, t}\right|^{2} \leq \frac{N_{0} W_{k}^{j}\left(2^{\frac{R_{k}^{j, t}}{W_{k}^{j}}}-1\right)}{\alpha_{k}^{j} p_{k}^{j}}\right) \\
& =F_{E(1)}\left(\frac{N_{0} W_{k}^{j}}{\alpha_{k}^{j} p_{k}^{j}}\left(2^{\frac{R_{k}^{j, t}}{W_{k}^{j}}}-1\right)\right),
\end{array}\right)
$$

where $F_{E(1)}$ represents cumulative distribution function of the exponentially distributed random variable whose mean value is 1. Assuming that $F_{E(1)}^{-1}$ is the inverse function of $F_{E(1)}$, the evaluated data rate $R_{k}^{j, t}$ can be expressed as [13]:

$$
R_{k}^{j, t}=W_{k}^{j} \log _{2}\left(1+\frac{\alpha_{k}^{j} p_{k}^{j} F_{E(1)}^{-1}(\varepsilon)}{N_{0} W_{k}^{j}}\right),
$$

while on the right side of this equation, all of the parameters have no relationship with slot index $t$. It inspires us to use (9) as an approximation of $\bar{R}_{k}^{j}$. Utilizing (6) and (9), the original problem is transformed into the following problem as:

$$
\begin{array}{ll}
\max _{\mathbf{m}, \mathbf{p}} & \sum_{j=1}^{2} \sum_{k=1}^{K}\left\{\bar{R}_{k}^{j} m_{k}^{j}-q\left(p_{k}^{j} m_{k}^{j}+2 P_{\text {cir }}\right)\right\} \\
\text { s.t. } & \sum_{j=1}^{2} W_{k}^{j} \log _{2}\left(1+\frac{\alpha_{k}^{j} p_{k}^{j} F_{E(1)}^{-1}(\varepsilon)}{N_{0} W_{k}^{j}}\right) m_{k}^{j} T \geq B, \quad \forall k,
\end{array}
$$$$
(6 d) \sim(6 f) .
$$

\section{B. Proposed Iterative Algorithm}

Based on the aforementioned analysis, it is clear that (10) is a non-convex combinatorial problem. To solve this problem, we propose an iterative EE-PRA algorithm (Algorithm 1) on the basis of Dinkelbach method [14]. The algorithm includes two layers. The outer layer is used to update $q_{t}$ until it reaches the optimal energy efficiency. The inner layer is used to find the solution $\{\mathbf{m}, \mathbf{p}\}$ of (10) with a constant parameter $q_{t}$.

\section{1) Outer Layer to Update $q_{t}$}

As mentioned in Section III-A, we transform the fractional objective function into a subtractive form, the outer layer optimization problem can be summarized as:

$$
\min _{q} F(q)=\max _{\mathbf{m}, \mathbf{p}} \sum_{j=1}^{2} \sum_{k=1}^{K}\left\{\bar{R}_{k}^{j} m_{k}^{j}-q\left(p_{k}^{j} m_{k}^{j}+2 P_{\text {cir }}\right)\right\}
$$

$$
\text { s.t. } \quad(6 f) \sim(6 f),(10 b) .
$$

We adopt Dinkelbach method [14] to solve (11), which can generate a converged sequence of $q_{t}$. The details are shown in Line 3 and Line $11 \sim 13$ of Algorithm 1 . 


\section{2) Inner Layer with Constant Parameter $q_{t}$}

The inner layer is used to find the solution $\left\{\mathbf{m}_{t}, \mathbf{p}_{t}\right\}$ of (10). In each iteration, we decouple (10) into two subproblems and $\left\{\mathbf{m}_{t}, \mathbf{p}_{t}\right\}$ is optimized with the constant parameter $q_{t}$.

Firstly, the power control vector $\mathbf{p}_{t}$ is fixed and (10) can be converted into a linear programming problem (12) with respect to variable $\mathbf{m}$ as:

$$
\begin{aligned}
\max _{\mathbf{m}} & \left\{\sum_{j=1}^{2} \sum_{k=1}^{K}\left(\bar{R}_{k}^{j}-q p_{k}^{j}\right) m_{k}^{j}\right\}-2 q P_{c i r} \\
\text { s.t. } \quad & \sum_{j=1}^{2} W_{k}^{j} \log _{2}\left(1+\frac{\alpha_{k}^{j} p_{k}^{j} F_{E(1)}^{-1}(\varepsilon)}{N_{0} W_{k}^{j}}\right) m_{k}^{j} T \geq B, \forall k \\
& \sum_{k=1}^{K} m_{k}^{1} \leq 1, \\
& 0 \leq m_{k}^{j} \leq 1 \quad \forall j, \quad \forall k \in \mathcal{K}
\end{aligned}
$$

which can be solved through interior point method.

Secondly, substituting time allocation solution $\mathbf{m}^{*}$ of (12) into (10), (10) can be converted into a convex problem with variable $\mathbf{p}$ as:

$$
\begin{aligned}
& \max _{\mathbf{p}}\left\{\sum_{j=1}^{2} \sum_{k=1}^{K}\left(\bar{R}_{k}^{j}-q p_{k}^{j}\right) m_{k}^{j *}\right\}-2 q P_{c i r} \\
& \text { s.t. } \sum_{j=1}^{2} W_{k}^{j} \log _{2}\left(1+\frac{\alpha_{k}^{j} p_{k}^{j} F_{E(1)}^{-1}(\varepsilon)}{N_{0} W_{k}^{j}}\right) m_{k}^{j *} T \geq B, \forall k \\
& 0 \leq p_{k}^{j} \leq \frac{W_{k}^{j}}{W_{\max }} P_{\max } \quad \forall j=1,2, \forall k \in \mathcal{K}_{\mathcal{L}} \cup \mathcal{K}_{\mathcal{H}}
\end{aligned}
$$

The Lagrange function of (13) is:

$$
\begin{aligned}
\mathcal{L}(\mathbf{p}, \boldsymbol{\beta}) & =\sum_{j=1}^{2} \sum_{k=1}^{K}\left\{\left(1+\beta_{k} T\right) m_{k}^{j *} \bar{R}_{k}^{j}-q m_{k}^{j *} p_{k}^{j}\right\} \\
& -\sum_{k=1}^{K} \beta_{k} B-q M P_{c i r},
\end{aligned}
$$

where $\boldsymbol{\beta}$ is the Lagrange multiplier vector of constraint (13b) with elements $\beta_{k} \geq 0, k \in\{1, \ldots, K\}$. Considering that power control policies are independent among different VUEs and different phases, we can use Lagrange dual decomposition theory to find a solution of (13). The Lagrange function can be decomposed as:

$$
\mathcal{L}(\mathbf{p}, \boldsymbol{\beta})=\sum_{j=1}^{2} \sum_{k=1}^{K} \mathcal{L}_{k}^{j}\left(p_{k}^{j}\right)-\sum_{k=1}^{K} \beta_{k} B-2 q P_{\text {cir }},
$$

where $\mathcal{L}_{k}^{j}\left(p_{k}^{j}\right)=\left(1+\beta_{k} T\right) m_{k}^{j *} \bar{R}_{k}^{j}-q m_{k}^{j *} p_{k}^{j}$. To find the maximal value of $\mathcal{L}_{k}^{j}\left(p_{k}^{j}\right)$, we can get its derivative about $p_{k}^{j}$ :

$$
\frac{\partial\left(\mathcal{L}_{k}^{j}\right)}{\partial\left(p_{k}^{j}\right)}=\left(1+\beta_{k} T\right) m_{k}^{j} W_{k}^{j} \frac{\alpha_{k}^{j} F_{E(1)}^{-1}(\varepsilon)}{N_{0} W_{k}^{j}+\alpha_{k}^{j} p_{k}^{j} F_{E(1)}^{-1}(\varepsilon)}-q m_{k}^{j} .
$$

According to the Karush-Kuhn-Tucker (KKT) conditions, the closed-form power control policy is:

$$
p_{k}^{j}=\left\{\begin{array}{ll}
{\left[\frac{\left(1+\beta_{k} T\right) W_{k}^{j}}{\ln 2 \cdot q}-\frac{N_{0} W_{k}^{j}}{\alpha_{k}^{j} F_{E(1)}^{-1}(\varepsilon)}\right]_{0}^{\frac{W_{k}^{j}}{W_{\max }} P_{\max }},} & m_{k}^{j} \neq 0 \\
0, & m_{k}^{j}=0
\end{array},\right.
$$

and $[x]_{a}^{b}=x$, if $a \leq x \leq b$. Furthermore, the dual problem of the original problem (13) can be obtained as:

$$
\begin{aligned}
& \min _{\boldsymbol{\beta}} \mathcal{G}(\boldsymbol{\beta})=\max _{\mathbf{p}} \mathcal{L}(\mathbf{p}, \boldsymbol{\beta}), \\
& \text { s.t. } \quad \beta_{k} \geq 0 \quad \forall k \in \mathcal{K}_{\mathcal{L}} \cup \mathcal{K}_{\mathcal{H}} .
\end{aligned}
$$

Since the dual function in (18) is differentiable, the Lagrange multiplier $\boldsymbol{\beta}$ can be updated by subgradient method [15]:

$$
\beta_{\mathrm{k}}(t+1)=\left[\beta_{k}(t)-\theta_{\beta}(t)\left(\sum_{j=1}^{2} m_{k}^{j *} T \bar{R}_{k}^{j}-B\right)\right]^{+} .
$$

Hence, the complexity of Algorithm 1 is $O\left(N_{\text {out }}\left(\theta_{1}^{\frac{1}{2}}\left(\theta_{1}+\theta_{2}\right) \theta_{2}^{2}+2 K^{2} N_{\text {sub }}\right)\right)$, where $N_{\text {out }}$ and $N_{s u b}$ are the iterations for the convergence of Dinkelbach and subgradient methods, respectively. And $O\left(\theta_{1}^{\frac{1}{2}}\left(\theta_{1}+\theta_{2}\right) \theta_{2}^{2}\right)$ is the complexity of interior point method with $\theta_{1}$ represents the number of inequality constraints and $\theta_{2}$ represents the number of variables. Besides, data analysis was performed before resource allocation and the analysis results are viewed as prior knowledge which have minor impact on algorithm complexity.

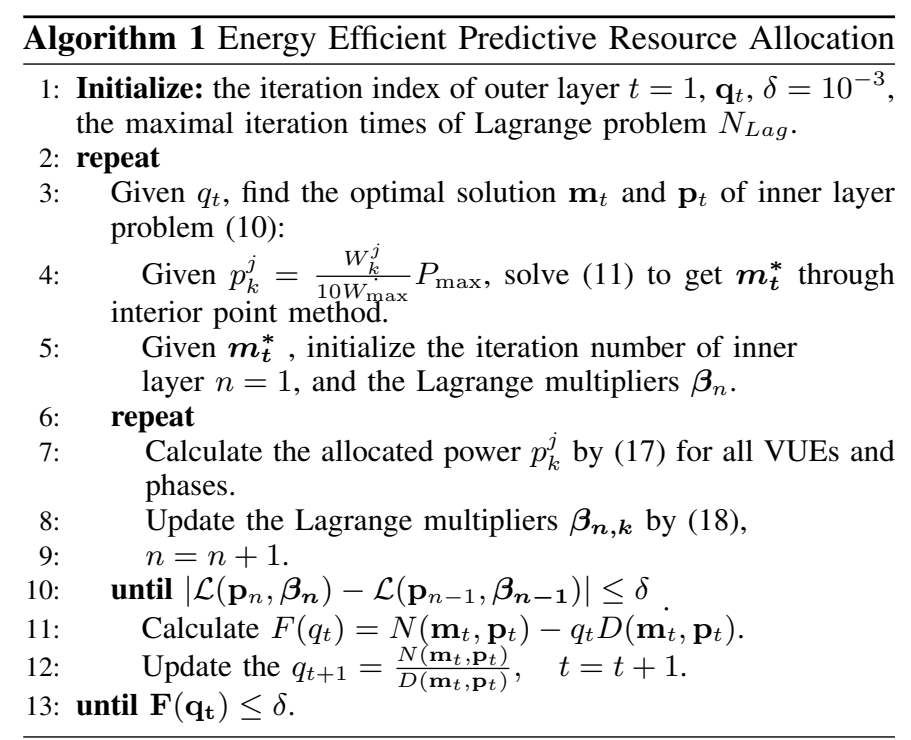


TABLE I

K-S TEST RESULT OF THE FITTED FUNCTION

\begin{tabular}{|c|c|c|}
\hline Fitted Function & Statistic D & p-value \\
\hline Exponential distribution & 0.6646 & 0 \\
\hline Normal distribution & 0.7666 & 0 \\
\hline KDE-guassian & 0.0114 & 0.4672 \\
\hline
\end{tabular}

${ }^{*}$ p-value $<0.05$ means the original hypothesis is rejected

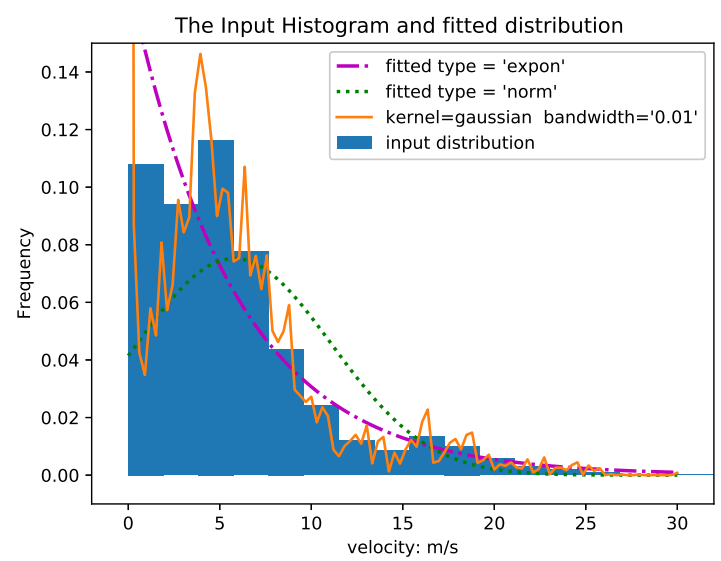

Fig. 2. The comparision between original data and fitted function.

\section{Simulation AND ANALYSis}

In this section, we present simulation parameters and data analysis results of speed distribution on T-drive dataset. Then we evaluate performance of the proposed EE-PRA scheme with numerical simulations.

\section{A. Simulation Parameters and Data Analysis Results}

As we depicted in Fig. 1, each BS covers the area of radius $R_{b}=250 \mathrm{~m}$, the maximum transmit power $P_{\max }=40 \mathrm{~W}$ and the maximum bandwidth $W_{\max }=10 \mathrm{MHz}$. On the condition that the available bandwidth in $\mathrm{BS}_{1}$ is more than that in $\mathrm{BS}_{2}$, we set $W_{1}=10 \mathrm{MHz}$ and $W_{2}=5 \mathrm{MHz}$. The path loss is $35.3+37.6 \cdot \log _{10}(d)$, where $d$ is the distance between VUE and BS in meter [8]. The standard derivation of shadowing is 8 $\mathrm{dB}$ [5], and the noise power spectral density $N_{0}$ is set to -114 $\mathrm{dBm} / \mathrm{MHz}$. In this paper, each VUE requests at least $B=$ 10 Mbits that needs to be transmitted within the prediction window with tolerable $\mathrm{OP} \varepsilon=0.1$. The prediction window is $10 \mathrm{~s}$ with the duration of one phase $T=5 \mathrm{~s}$, and the number of time slots in one phase is $N_{s}=50$. To explore the impact of VUE mobility on PRA, we consider the following two mobility patterns:

1) Model-Driven Mobility Pattern: In this pattern, the car-follow model [16] is used to model the mobility of HUEs. The LUEs move in a variable acceleration motion whose speed follows gaussian distribution of $0 \sim 5 \mathrm{~m} / \mathrm{s}$ and acceleration follows gaussian distribution of $0 \sim 0.05 \mathrm{~m} / \mathrm{s}^{2}$. This pattern is used to generate the speed of VUE with specific number of HUEs and LUEs.

2) Data-Driven Mobility Pattern: In this pattern, the speeds of VUE are generated according to the speed distribution analysis of dataset. As introduced in Section II, we test the estimated function by kolmogorov-smirnov test ( $K$-S test),

the results of which are shown in Table I. It is obvious that

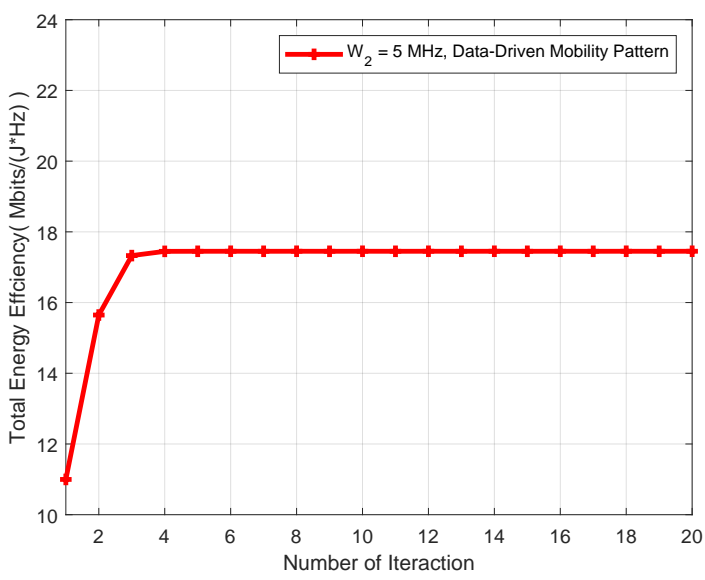

Fig. 3. The convergence of the proposed algorithm.

parameter estimation cannot describe the original distribution well. While KDE-gaussian with kernel width 0.01 can accept the original hypothesis that the fitted function has the same distribution as original data. Therefore, we use KDE-gaussian function to generate VUE speed. Fig. 2 shows a comparison between speed histogram and fitted function.

\section{B. Convergence Performance}

To evaluate convergence performance of the proposed EEPRA algorithm, simulations are performed as illustrated in Fig. 3. The speeds of $K=8$ VUEs is generated by data-driven mobility pattern. We can observe that the proposed scheme converges after about four iterations, which indicates that our proposed algorithm has a fast convergence speed.

\section{System Performance}

To evaluate the performance of the proposed scheme, the following three schemes are included as baseline schemes.

1) Equal Resource Allocation: In this non-predictive RA scheme, the time resource is evenly allocated among VUEs. In each time slot, BS serves VUE who has the largest instantaneous channel gain in the corresponding candidate set. If the sum of transmission time of VUE $k$ is greater than average transmission time (i.e., $\frac{2 N_{s}}{N_{U E}}$ ), we remove the VUE $k$ from the candidate set.

2) Random Resource Allocation: In this non-predictive RA scheme, the time and energy resources are randomly allocated until the data transmission completed.

3) EE-PRA with Predcition Error: To show the impact of prediction error on PRA, the prediction errors of available bandwidth and large scale channel gain are modeled as Gaussian distributed random variables with standard derivation 1 and 0.5 , respectively.

To evaluate EE performance under different speed distributions of VUE, simulations are performed in Fig. 4. The total number of VUEs $\mathcal{K}=8$, the number of HUEs and LUEs is not equal under different speed distribution levels. In 'High Mobility=Low Mobility' level, the number of HUEs is equal to that of LUEs. Similarly, 'High Mobility $\geq$ Low Mobility' refers to that there are more HUEs than LUEs. In ' $K D E$ ' level where the speed of VUE is generated by KDE-gaussian function, the number of HUEs is less than that of LUEs. For 


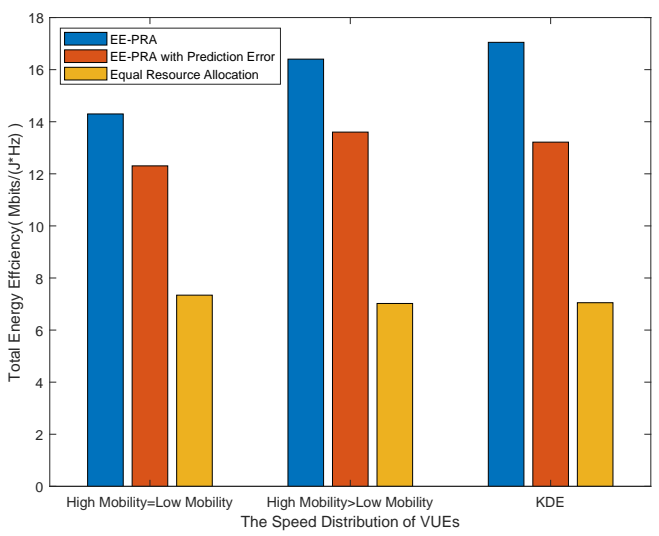

Fig. 4. The system performance under different speed distribution of VUEs.

fair comparison, all of the VUEs move at a constant speed. From Fig. 4, it is clear that the proposed EE-PRA scheme has significant gains over conventional scheme. Meanwhile, comparing the performance of the proposed scheme under different speed distributions, we observe that system EE of PRA increases when the number of HUEs is not equal to LUEs. So considering the different mobility patterns in PRA is necessary.

Fig. 5 shows the system EE of the proposed EE-PRA scheme and three baseline schemes versus the number of VUEs whose speeds are generated by model-driven mobility pattern. From the Fig. 5, we can observe that our proposed EEPRA scheme outperforms the other three baseline schemes in terms of system EE. The system EE first rises as the resource can be fully utilized as the number of VUE increases. When $K>8$, the system EE cannot be further improved due to limited resources. At last, the two PRA schemes, including EEPRA and EE-PRA with prediction error, perform better than non-predictive schemes attributed to the traffic prediction and the cooperation of neighboring BSs. Moreover, as the number of VUEs rises, the system EE of all four schemes rises first and then flattens out.

\section{CONCLUSIONS}

In this paper, we focused on the EE problem under the scenario of IoV. On the basis of real world dataset, we adopted a KDE method to characterize the distribution of vehicle's speed and used this information as priori knowledge to formulate a resource allocation problem. The formulated EE maximization problem was transformed using FP and CDI. Then, an iterative energy efficient predictive time and energy allocation scheme was proposed. Simulation results demonstrated that the proposed scheme exhibits dramatic gain over non-predictive resource allocation schemes even with prediction errors exist.

\section{ACKNOWLEDGMENT}

This work was supported in part by Project of International Cooperation and Exchanges NSFC under Grant No. 61860206005 , and in part by the National Natural Science Foundation of China under Grant No. 61671278.

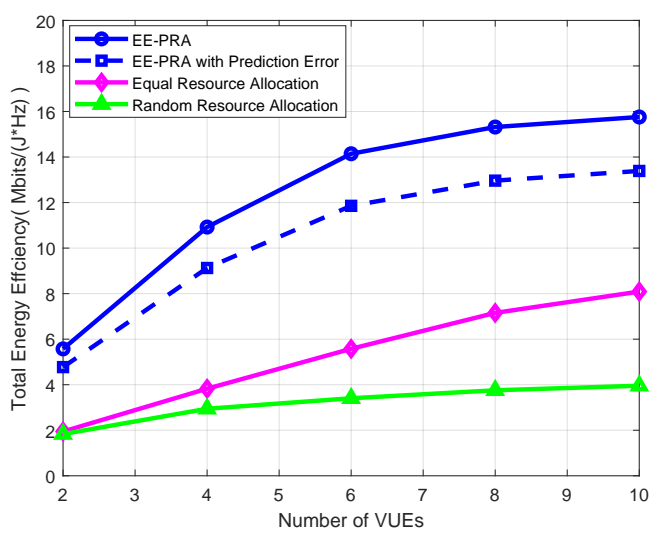

Fig. 5. The EE performance comparison of four schemes versus different number of VUEs.

\section{REFERENCES}

[1] H. Peng, L. Liang, X. Shen, and G. Y. Li, "Vehicular communications: A network layer perspective," IEEE Trans. Veh. Technol., vol. 68, no. 2, pp. 1064-1078, 2018.

[2] A. Nadembega, A. Hafid, and T. Taleb, "A destination and mobility path prediction scheme for mobile networks," IEEE Trans. Veh. Technol., vol. 64, no. 6, pp. 2577-2590, 2014.

[3] C. Zhang, H. Zhang, D. Yuan, and M. Zhang, "Citywide cellular traffic prediction based on densely connected convolutional neural networks," IEEE Commun. Lett., vol. 22, no. 8, pp. 1656-1659, 2018.

[4] C. Zhang, H. Zhang, J. Qiao, D. Yuan, and M. Zhang, "Deep transfer learning for intelligent cellular traffic prediction based on cross-domain big data," IEEE J. Sel. Areas Commun., vol. 37, no. 6, pp. 1389-1401, 2019.

[5] J. Guo, C. She, and C. Yang, "Predictive resource allocation with coarsegrained mobility pattern and traffic load information," in 2018 IEEE International Conference on Communications (ICC), 2018, pp. 1-6.

[6] M. Chen, L. Wang, J. Chen, X. Wei, and L. Lei, "A computing and content delivery network in the smart city: Scenario, framework, and analysis," IEEE Netw., vol. 33, no. 2, pp. 89-95, 2019.

[7] H. Abou-zeid, H. S. Hassanein, and S. Valentin, "Energy-efficient adaptive video transmission: Exploiting rate predictions in wireless networks," IEEE Trans. Veh. Technol., vol. 63, no. 5, pp. 2013-2026, 2014.

[8] C. She and C. Yang, "Energy efficient resource allocation for hybrid services with future channel gains," IEEE Transactions on Green Communications and Networking, vol. 4, no. 1, pp. 165-179, 2019.

[9] R. Atawia, H. S. Hassanein, H. Abou-Zeid, and A. Noureldin, "Robust content delivery and uncertainty tracking in predictive wireless networks," IEEE Trans. Wireless Commun., vol. 16, no. 4, pp. 2327-2339, 2017.

[10] J. Yuan, Y. Zheng, C. Zhang, W. Xie, X. Xie, G. Sun, and Y. Huang, "Tdrive: driving directions based on taxi trajectories," in Proceedings of the 18th SIGSPATIAL International conference on advances in geographic information systems, 2010, pp. 99-108.

[11] V. A. Epanechnikov, "Non-parametric estimation of a multivariate probability density," Theory of Probability \& Its Applications, vol. 14, no. 1, pp. 153-158, 1969.

[12] Y. Zhang, J. An, K. Yang, X. Gao, and J. Wu, "Energy-efficient user scheduling and power control for multi-cell OFDMA networks based on channel distribution information," IEEE Trans. Signal Process., vol. 66, no. 22, pp. 5848-5861, 2018.

[13] N. Mokari, K. Navaie, and M. G. Khoshkholgh, "Downlink radio resource allocation in ofdma spectrum sharing environment with partial channel state information," IEEE Trans. Wireless Commun., vol. 10, no. 10 , pp. 3482-3495, 2011.

[14] W. Dinkelbach, "On nonlinear fractional programming," Management science, vol. 13, no. 7, pp. 492-498, 1967.

[15] S. Boyd, S. P. Boyd, and L. Vandenberghe, Convex optimization. Cambridge university press, 2004.

[16] H. Zhou, B. Liu, T. H. Luan, F. Hou, L. Gui, Y. Li, Q. Yu, and X. Shen, "Chaincluster: Engineering a cooperative content distribution framework for highway vehicular communications," IEEE Trans. Intell. Transp. Syst., vol. 15, no. 6, pp. 2644-2657, 2014. 